November, 2009

\title{
The Fayet-Iliopoulos term and nonlinear self-duality
}

\author{
Sergei M. Kuzenko 1 \\ School of Physics M013, The University of Western Australia \\ 35 Stirling Highway, Crawley W.A. 6009, Australia
}

\begin{abstract}
The $\mathcal{N}=1$ supersymmetric Born-Infeld action is known to describe the vector Goldstone multiplet for partially broken $\mathcal{N}=2$ rigid supersymmetry, and this model is believed to be unique. However, it can be deformed by adding the FayetIliopoulos term without losing the second nonlinearly realized supersymmetry. Although the first supersymmetry then becomes spontaneously broken, the deformed action still describes partial $\mathcal{N}=2 \rightarrow \mathcal{N}=1$ supersymmetry breaking. The unbroken supercharges in this theory correspond to a different choice of $\mathcal{N}=1$ subspace in the $\mathcal{N}=2$ superspace, as compared with the undeformed case. Implications of the Fayet-Iliopoulos term for general models for self-dual nonlinear supersymmetric electrodynamics are discussed. The known ubiquitous appearance of the Volkov-Akulov action in such models is explained. We also present a two-parameter duality-covariant deformation of the $\mathcal{N}=1$ supersymmetric Born-Infeld action as a model for partial breaking of $\mathcal{N}=2$ supersymmetry.
\end{abstract}

\footnotetext{
${ }^{1}$ kuzenko@cyllene.uwa.edu.au
} 
Recently, there have been interesting discussions [1, 2] of the supercurrent [3] (i.e. the multiplet of currents containing the energy-momentum tensor and the supersymmetry current) in $\mathcal{N}=1$ supersymmetric gauge theories with a Fayet-Iliopoulos (FI) term [4]. These works are primarily targeted at phenomenological applications of supergravity theories. In the present note, we would like to elaborate on somewhat different and more formal aspects such as implications of the FI term for partial supersymmetry breaking, and more generally in the context of models for self-dual nonlinear supersymmetric electrodynamics. In particular, we will revisit the conclusion of [5, 6] about uniqueness of the Goldstone-Maxwell multiplet model for partially broken $\mathcal{N}=2$ supersymmetry. At the end of this note, we will also comment on the claim made in [2] that "no supercurrent supermultiplet exists for globally supersymmetric gauge theories with non-zero Fayet-Iliopoulos terms."

The $\mathcal{N}=1$ supersymmetric Born-Infeld (BI) action is 1

$$
\begin{aligned}
S_{\mathrm{SBI}}[W] & =\frac{1}{4} \int \mathrm{d}^{6} z W^{2}+\frac{1}{4} \int \mathrm{d}^{6} \bar{z} \bar{W}^{2}+\frac{g^{2}}{4} \int \mathrm{d}^{8} z \frac{W^{2} \bar{W}^{2}}{1+\frac{1}{2} A+\sqrt{1+A+\frac{1}{4} B^{2}}}, \\
A & =\frac{g^{2}}{8}\left(D^{2} W^{2}+\bar{D}^{2} \bar{W}^{2}\right), \quad B=\frac{g^{2}}{8}\left(D^{2} W^{2}-\bar{D}^{2} \bar{W}^{2}\right) .
\end{aligned}
$$

Here $g$ is the coupling constant, $W_{\alpha}$ the chiral field strength of an Abelian vector multiplet,

$$
W_{\alpha}=-\frac{1}{4} \bar{D}^{2} D_{\alpha} V, \quad V=\bar{V},
$$

with $V$ the gauge prepotential. The action (11) was introduced for the first time in Refs. [9, 10] as a supersymmetric extension of the BI theory [11], and as such it is not unique. Bagger and Galperin [5], and later Roček and Tseytlin [6], using alternative techniques, discovered that $S_{\mathrm{SBI}}$ is the action for a Goldstone-Maxwell multiplet associated with $\mathcal{N}=2 \rightarrow \mathcal{N}=1$ partial supersymmetry breaking. This action was argued to be unique [5, 6]. Being manifestly $\mathcal{N}=1$ supersymmetric, $S_{\mathrm{SBI}}$ also proves to be invariant under a second, nonlinearly realized supersymmetry transformation

$$
\delta W_{\alpha}=\eta_{\alpha}+\frac{g^{2}}{4}\left(\frac{1}{4} \bar{D}^{2} \bar{X} \eta_{\alpha}+\mathrm{i} \partial_{\alpha \dot{\alpha}} X \bar{\eta}^{\dot{\alpha}}\right)
$$

with $\eta_{\alpha}$ a constant spinor parameter. Here $X$ is a chiral superfield, $\bar{D}_{\dot{\alpha}} X=0$, satisfying the nonlinear constraint [5, 6]

$$
X+\frac{g^{2}}{16} X \bar{D}^{2} \bar{X}=W^{2}
$$

\footnotetext{
${ }^{1}$ We follow the notation and conventions adopted in [7, 8]. The superspace integration measures in (11) are defined as follows: $\mathrm{d}^{6} z:=\mathrm{d}^{4} x \mathrm{~d}^{2} \theta, \mathrm{d}^{6} \bar{z}:=\mathrm{d}^{4} x \mathrm{~d}^{2} \bar{\theta}$ and $\mathrm{d}^{8} z:=\mathrm{d}^{4} x \mathrm{~d}^{2} \theta \mathrm{d}^{2} \bar{\theta}$.
} 
The proof of the invariance is based on the observations [5, 6] that (i) the functional (11) can be rewritten in the form

$$
S_{\mathrm{SBI}}[W]=\frac{1}{4} \int \mathrm{d}^{6} z X+\frac{1}{4} \int \mathrm{d}^{6} \bar{z} \bar{X}
$$

and (ii) the chiral scalar $X$ transforms under (3) as

$$
\delta X=2 \eta^{\alpha} W_{\alpha}
$$

Consider now the $\mathcal{N}=1$ supersymmetric FI term [4]

$$
S_{\mathrm{FI}}=2 \xi \int \mathrm{d}^{8} z V=\frac{\xi}{2} \int \mathrm{d}^{6} z \theta^{\alpha} W_{\alpha}+\frac{\xi}{2} \int \mathrm{d}^{6} \bar{z} \bar{\theta}_{\dot{\alpha}} \bar{W}^{\dot{\alpha}}
$$

It is easy to see that $S_{\mathrm{FI}}$ is also invariant under the second nonlinearly realized supersymmetry (3), as pointed out recently in [12].2 Therefore, the theory with action

$$
S=S_{\mathrm{SBI}}[W]+S_{\mathrm{FI}}
$$

is manifestly $\mathcal{N}=1$ supersymmetric, and is invariant under the second nonlinearly realized supersymmetry (3). We are going to show that the deformed action also describes partial $\mathcal{N}=2 \rightarrow \mathcal{N}=1$ supersymmetry breaking.

Let us study the bosonic sector of the component Lagrangian. The component fields contained in $W_{\alpha}$ are:

$$
\begin{aligned}
\lambda_{\alpha}(x) & =\left.W_{\alpha}\right|_{\theta=0}, \\
F_{\alpha \beta}(x) & =-\left.\frac{\mathrm{i}}{4}\left(D_{\alpha} W_{\beta}+D_{\beta} W_{\alpha}\right)\right|_{\theta=0}, \\
\mathcal{D}(x) & =-\left.\frac{1}{2} D^{\alpha} W_{\alpha}\right|_{\theta=0},
\end{aligned}
$$

with

$$
F_{\alpha \dot{\alpha} \beta \dot{\beta}} \equiv\left(\sigma^{a}\right)_{\alpha \dot{\alpha}}\left(\sigma^{b}\right)_{\beta \dot{\beta}} F_{a b}=2 \varepsilon_{\alpha \beta} \bar{F}_{\dot{\alpha} \dot{\beta}}+2 \varepsilon_{\dot{\alpha} \dot{\beta}} F_{\alpha \beta}
$$

the electromagnetic field strength. Setting the photino to zero, $\lambda_{\alpha}=0$, the bosonic Lagrangian can be shown to be

$$
L_{\text {boson }}=\frac{1}{g^{2}}\left\{1-\sqrt{1+g^{2}(\boldsymbol{u}+\overline{\boldsymbol{u}})+\frac{1}{4} g^{4}(\boldsymbol{u}-\overline{\boldsymbol{u}})^{2}}\right\}+\xi \mathcal{D},
$$

\footnotetext{
${ }^{2}$ This property is directly related to the fact that that the $\mathcal{N}=1$ FI term preserves also $\mathcal{N}=2$ supersymmetry [13].
} 
where we have defined

$$
\boldsymbol{u}:=\left.\frac{1}{8} D^{2} W^{2}\right|_{\theta_{\alpha}=\lambda_{\alpha}=0}=\omega-\frac{1}{2} \mathcal{D}^{2}, \quad \omega:=\frac{1}{4} F^{a b} F_{a b}+\frac{\mathrm{i}}{4} F^{a b} \tilde{F}_{a b},
$$

with $\tilde{F}_{a b}$ the Hodge-dual of $F_{a b}$. From $L_{\text {boson }}$ we read off the equation of motion for the auxiliary field:

$$
\frac{\mathcal{D}}{\sqrt{1+g^{2}(\boldsymbol{u}+\overline{\boldsymbol{u}})+\frac{1}{4} g^{4}(\boldsymbol{u}-\overline{\boldsymbol{u}})^{2}}}=-\xi .
$$

Its solution is

$$
\mathcal{D}=-\frac{\xi}{\sqrt{1+g^{2} \xi^{2}}} \sqrt{1+g^{2}(\omega+\bar{\omega})+\frac{1}{4} g^{4}(\omega-\bar{\omega})^{2}} .
$$

Here the second factor on the right is essentially the BI Lagrangian [11]:

$$
\begin{aligned}
L_{\mathrm{BI}} & =\frac{1}{g^{2}}\left\{1-\sqrt{1+g^{2}(\omega+\bar{\omega})+\frac{1}{4} g^{4}(\omega-\bar{\omega})^{2}}\right\} \\
& =\frac{1}{g^{2}}\left\{1-\sqrt{-\operatorname{det}\left(\eta_{a b}+g F_{a b}\right)}\right\}
\end{aligned}
$$

Upon elimination of the auxiliary field, the bosonic Lagrangian (11) becomes

$$
L_{\text {boson }}=\frac{1}{g^{2}}\left\{1-\sqrt{1+g^{2} \xi^{2}} \sqrt{-\operatorname{det}\left(\eta_{a b}+g F_{a b}\right)}\right\} \text {. }
$$

Modulo an irrelevant constant term and an overall normalization factor, this is again the BI Lagrangian.

Looking at the expression for the auxiliary field, eq. (14), we see that it acquires a non-vanishing expectation value

$$
\langle\mathcal{D}\rangle=-\frac{\xi}{\sqrt{1+g^{2} \xi^{2}}}
$$

Therefore, the manifestly realized supersymmetry of the theory (8) becomes spontaneously broken. The corresponding supersymmetry transformation of the photino is now

$$
\delta \lambda_{\alpha}=\left.\mathrm{i}(\epsilon Q+\bar{\epsilon} \bar{Q}) W_{\alpha}\right|_{\theta=0}=\langle\mathcal{D}\rangle \epsilon_{\alpha}+\text { field-dependent terms }
$$

and therefore the photino turns into a Goldstino. As a result, the situation is now the following. The model under consideration, eq. (8), possesses two supersymmetries, of 
which one $(Q)$ is linearly realized, and the other $(S)$ is nonlinearly realized, as described by eq. (31). On the mass shell, both $Q$ - and $S$-supersymmetries become nonlinearly realized. Clearly, this does not mean that the $\mathcal{N}=2$ supersymmetry is completely broken, for there is only one Goldstino in the theory. Therefore, a special combination of the $Q$ - and $S$-supersymmetries must remain unbroken. This can be seen explicitly as follows. The $Q$ - and $S$-supersymmetry transformations of the theory (8) form the $\mathcal{N}=2$ super-Poincaré algebra without central charge,

$$
\begin{array}{rlrl}
\left\{Q_{\alpha}, \bar{Q}_{\dot{\beta}}\right\} & =2\left(\sigma^{c}\right)_{\alpha \dot{\beta}} P_{c}, & \left\{S_{\alpha}, \bar{S}_{\dot{\beta}}\right\}=2\left(\sigma^{c}\right)_{\alpha \dot{\beta}} P_{c} \\
\left\{Q_{\alpha}, S_{\beta}\right\}=0, & \left\{Q_{\alpha}, \bar{S}_{\dot{\beta}}\right\}=0
\end{array}
$$

see [5, 6] for more detail. Now, using the transformation laws (3) and (18), one can readily check that $\mathcal{N}=1$ supersymmetry transformations generated by

$$
\mathcal{Q}_{\alpha}:=\cos \varphi Q_{\alpha}+\sin \varphi S_{\alpha}, \quad \tan \varphi=\frac{\xi}{\sqrt{1+g^{2} \xi^{2}}}
$$

remain unbroken. We see that the result of adding the FI term to the $\mathcal{N}=1$ supersymmetric BI action amounts to a $\mathrm{U}(1)$ rotation of the unbroken $\mathcal{N}=1$ supersymmetry generators in the $\mathcal{N}=2$ super-Poincaré algebra. Therefore, the dynamical system (8) is a (deformed) vector Goldstone multiplet model for partial supersymmetry breaking $\mathcal{N}=2 \rightarrow \mathcal{N}=1$. Similarly to the considerations in [6, 12, the action (86) can be obtained by (i) starting from a non-renormalized model for an Abelian $\mathcal{N}=2$ vector multiplet with two types (electric and magnetic) of $\mathcal{N}=2$ FI terms [14, 15], and then (ii) integrating out a massive $\mathcal{N}=1$ scalar multiplet 3

The supersymmetric BI theory (1) is known to be invariant under $\mathrm{U}(1)$ duality rotations [17, 18]. It is in fact a special member of the family of models for self-dual nonlinear $\mathcal{N}=1$ supersymmetric electrodynamics constructed in [18] and described by actions of the form:

$$
S[W]=\frac{1}{4} \int \mathrm{d}^{6} z W^{2}+\frac{1}{4} \int \mathrm{d}^{6} \bar{z} \bar{W}^{2}+\frac{1}{4} \int \mathrm{d}^{8} z W^{2} \bar{W}^{2} \Lambda(u, \bar{u}), \quad u:=\frac{1}{8} D^{2} W^{2},
$$

where $\Lambda(u, \bar{u})$ is a real analytic function of one complex variable. In accordance with [18], this theory possesses $\mathrm{U}(1)$ duality invariance provided $\Lambda$ obeys the self-duality equation

$$
\operatorname{Im}\left\{\Gamma-\bar{u} \Gamma^{2}\right\}=0, \quad \Gamma(u, \bar{u}):=\frac{\partial}{\partial u}(u \Lambda(u, \bar{u}))
$$

\footnotetext{
${ }^{3}$ It would be interesting to understand how to integrate out massive degrees of freedom in the nonAbelian extensions [16] of the Antoniadis-Partouche-Taylor model [14].
} 
The fermionic sector of such models turns out to possess a remarkable structure [19].

As demonstrated in [19], under the only additional restriction

$$
\Lambda_{u \bar{u}}(0,0)=3 \Lambda^{3}(0,0)
$$

the component fermionic action coincides, modulo a nonlinear field redefinition, with the Volkov-Akulov action [20] 4. At first sight, this ubiquity of the Goldstino action in the framework of nonlinear self-duality looks somewhat miraculous. It can be explained, however, if we let the FI term enter the game and consider the following model

$$
S[W]+S_{\mathrm{FI}}
$$

In the purely bosonic sector, the equation of motion for the auxiliary field is

$$
\mathcal{D}[1-\overline{\boldsymbol{u}} \Gamma(\boldsymbol{u}, \overline{\boldsymbol{u}})-\boldsymbol{u} \bar{\Gamma}(\boldsymbol{u}, \overline{\boldsymbol{u}})]=-\xi,
$$

with $\boldsymbol{u}$ defined as in (12). This equation should be used to express $\mathcal{D}$ in terms of the electromagnetic field strength, $\mathcal{D}=f(\omega, \bar{\omega})$. Generically, the auxiliary field develops a non-vanishing expectation value, $\langle\mathcal{D}\rangle \neq 0$, which must satisfy an algebraic nonlinear equation that follows from (25) by setting $\omega=0.5$ As a result, the supersymmetry becomes spontaneously broken, and thus the photino action should be related to the Goldstino action, due to the unique of the latter.

It is worth briefly recalling the structure of the bosonic sector in the model (21). The corresponding equation of motion for $\mathcal{D}$ is obtained from (25) by setting $\xi=0$, and hence it always has the solution $\mathcal{D}=0$. With this solution chosen, the dynamics of the electromagnetic field is described by the Lagrangian

$$
L=-\frac{1}{2}(\omega+\bar{\omega})+\omega \bar{\omega} \Lambda(\omega, \bar{\omega})
$$

with the interaction $\Lambda$ obeying the self-duality equation (22). This is a model for self-dual nonlinear electrodynamics in the sense of [22, 23, 24], of which the BI theory (15) is a special case. Such theories and their generalizations possess very interesting properties, see, e.g., the second reference in [18] and [25] for reviews. It is natural to ask the following question: Is self-duality preserved in some form in the case of deformed theory (24) with a non-zero $\mathcal{D}$ obeying the equation (25)? We now turn to answering this question.

The model (21) can be generalized to include couplings to supermultiplets containing the dilaton and axion, the NS and RR two-forms, $B_{2}$ and $C_{2}$, and the RR four-form, $C_{4}$,

\footnotetext{
${ }^{4}$ The results in 19 extended the earlier component analysis 21 of the supersymmetric BI theory (1).

${ }^{5}$ In some cases, the algebraic nonlinear equation on $\langle\mathcal{D}\rangle$ has no solution, and then (24) is inconsistent.
} 
as presented in [18] building on the bosonic constructions of [26, 27, 28]. The extended action has the form

$$
S[W, \Phi, \beta, \gamma, \Omega]=S[\mathbb{W}, \Phi]+\left\{\int \mathrm{d}^{6} z\left(\Omega+\frac{1}{2} \gamma^{\alpha} \mathbb{W}_{\alpha}\right)+\text { c.c. }\right\},
$$

where

$$
\begin{aligned}
S[W, \Phi] & =\frac{\mathrm{i}}{4} \int \mathrm{d}^{6} z \Phi W^{2}-\frac{\mathrm{i}}{4} \int \mathrm{d}^{6} \bar{z} \bar{\Phi} \bar{W}^{2} \\
& -\frac{1}{16} \int \mathrm{d}^{8} z(\Phi-\bar{\Phi})^{2} W^{2} \bar{W}^{2} \Lambda\left(\frac{\mathrm{i}}{16}(\Phi-\bar{\Phi}) D^{2} W^{2}, \frac{\mathrm{i}}{16}(\Phi-\bar{\Phi}) \bar{D}^{2} \bar{W}^{2}\right)
\end{aligned}
$$

describes $\operatorname{SL}(2, \mathbb{R})$ duality invariant coupling of the vector multiplet to the dilaton-axion chiral multiplet $\Phi$, and

$$
\mathbb{W}_{\alpha}:=W_{\alpha}+\mathrm{i} \beta_{\alpha}
$$

is the supersymmetrization of $F+B$. Here $\beta_{\alpha}, \gamma_{\alpha}$ and $\Omega$ are unconstrained chiral superfields which include, among their components, the fields $B_{2}, C_{2}$ and $C_{4}$, respectively. The resulting action is invariant under the following gauge transformations:

$$
\begin{gathered}
\delta \beta_{\alpha}=\mathrm{i} \delta W_{\alpha}=\mathrm{i} \bar{D}^{2} D_{\alpha} K_{1}, \\
\delta \gamma_{\alpha}=\mathrm{i} \bar{D}^{2} D_{\alpha} K_{2}, \quad \delta \Omega=\frac{1}{2} \beta^{\alpha} \bar{D}^{2} D_{\alpha} K_{2}, \\
\delta \Omega=\mathrm{i} \bar{D}^{2} K_{3}
\end{gathered}
$$

with $K_{i}$ real unconstrained superfields. The action (27) reduces to (21) by setting $\Phi=-\mathrm{i}$ and switching off the other chiral superfields $\beta_{\alpha}, \gamma_{\alpha}$ and $\Omega$.

As demonstrated in [18, the theory (27) is invariant under $\operatorname{SL}(2, \mathbb{R})$ duality transformations

$$
\left(\begin{array}{l}
M_{\alpha}^{\prime} \\
W_{\alpha}^{\prime}
\end{array}\right)=\left(\begin{array}{ll}
a & b \\
c & d
\end{array}\right)\left(\begin{array}{l}
M_{\alpha} \\
W_{\alpha}
\end{array}\right), \quad \Phi^{\prime}=\frac{a \Phi+b}{c \Phi+d}, \quad\left(\begin{array}{ll}
a & b \\
c & d
\end{array}\right) \in \mathrm{SL}(2, \mathbb{R}),
$$

provided the superfields $\beta_{\alpha}, \gamma_{\alpha}$ and $\Omega$ transform as

$$
\begin{aligned}
\left(\begin{array}{l}
\gamma^{\prime} \\
\beta^{\prime}
\end{array}\right) & =\left(\begin{array}{ll}
a & b \\
c & d
\end{array}\right)\left(\begin{array}{l}
\gamma \\
\beta
\end{array}\right), \\
\Omega^{\prime} & =\Omega-\frac{\mathrm{i}}{4} b d \beta^{2}-\frac{\mathrm{i}}{2} b c \beta \gamma-\frac{\mathrm{i}}{4} a c \gamma^{2} .
\end{aligned}
$$

Here $M_{\alpha}$ denotes a variational derivative of the action with respect to the field strength,

$$
\text { i } M_{\alpha}:=2 \frac{\delta}{\delta W^{\alpha}} S[W, \Phi, \beta, \gamma, \Omega] \text {. }
$$


A detailed discussion can be found in [18].

Using the second form of the FI term, eq. (17), the action (24) is seen to be of the type (27) with the following "frozen" values for background fields: $\gamma_{\alpha}=\xi \theta_{\alpha}, \Phi=-\mathrm{i}$ and $\beta_{\alpha}=$ $\Omega=0$. As a natural generalization, an ansatz compatible with duality transformations is $\gamma_{\alpha} \propto \theta_{\alpha}, \beta_{\alpha} \propto \theta_{\alpha}$ and $\Omega \propto \theta^{2}$. A consistent with duality choice is

$$
\gamma_{\alpha}=\xi \theta_{\alpha}, \quad \beta_{\alpha}=\zeta \theta_{\alpha}, \quad \Omega=0, \quad \xi, \zeta \in \mathbb{R}
$$

As follows from (32b), applying a duality transformation generates a purely imaginary non-zero value for $\Omega$ which, however, does not contribute to the action.

Let us now return to the model (24) and consider its duality-covariant extension

$$
S[\mathcal{W}]+S_{\mathrm{FI}}, \quad \mathcal{W}_{\alpha}:=W_{\alpha}+\mathrm{i} \zeta \theta_{\alpha}
$$

Here the deformed field strength $\mathcal{W}_{\alpha}$ obeys the modified Bianchi identity

$$
\bar{D}_{\dot{\alpha}} \overline{\mathcal{W}}^{\dot{\alpha}}-D^{\alpha} \mathcal{W}_{\alpha}=4 \mathrm{i} \zeta
$$

This action is invariant under inhomogeneous supersymmetry transformations

$$
\delta W_{\alpha}=-\mathrm{i} \zeta \epsilon_{\alpha}+\mathrm{i}(\epsilon Q+\bar{\epsilon} \bar{Q}) W_{\alpha}
$$

If $S[W]$ coincides with the supersymmetric BI action (11), then the resulting model

$$
S_{\mathrm{SBI}}[\mathcal{W}]+S_{\mathrm{FI}}
$$

is also invariant under a second nonlinearly realized supersymmetry, which is a natural generalization of (3),

$$
\delta W_{\alpha}=\eta_{\alpha}+\frac{g^{2}}{4}\left(\frac{1}{4} \bar{D}^{2} \overline{\mathcal{X}} \eta_{\alpha}+\mathrm{i} \partial_{\alpha \dot{\alpha}} \mathcal{X} \bar{\eta}^{\dot{\alpha}}\right)
$$

where $\mathcal{X}$ is a chiral superfield, $\bar{D}_{\dot{\alpha}} \mathcal{X}=0$, satisfying the nonlinear constraint

$$
\mathcal{X}+\frac{g^{2}}{16} \mathcal{X} \bar{D}^{2} \overline{\mathcal{X}}=\mathcal{W}^{2}
$$

compare with (4). Our action (38) is a two-parameter deformation of the supersymmetric BI theory (1). This action appears to be the most general Goldstone-Maxwell multiplet model for partial $\mathcal{N}=2 \rightarrow \mathcal{N}=1$ supersymmetry breaking. Requiring the first supersymmetry to be manifest eliminates one of the deformations, $\zeta=0$. The supersymmetric $\mathrm{BI}$ action (11) is indeed unique if the first supersymmetry is required to be unbroken. 
In conclusion, we would like to comment on the statement made in [2] that "no supercurrent supermultiplet exists for globally supersymmetric gauge theories with non-zero Fayet-Iliopoulos terms." A general scheme to compute supercurrents [3] in rigid supersymmetric theories is by evaluating a variational derivative of the action functional with respect to the gravitational superfield $H^{\alpha \dot{\alpha}}$,

$$
J_{\alpha \dot{\alpha}}=\frac{\Delta S}{\Delta H^{\alpha \dot{\alpha}}},
$$

with the idea due to Ogievetsky and Sokatchev [29]. More specifically, the procedure is as follows: (i) one should lift the theory to a curved superspace corresponding to one of the known off-shell supergravity formulations (realized in terms of the gravitational superfield $H^{\alpha \dot{\alpha}}$ and an appropriate compensator); (ii) compute the (covariantized) variational derivative $\Delta S / \Delta H^{\alpha \dot{\alpha}}$; (iii) return to the flat superspace by switching off the supergravity prepotentials. The scheme is worked out in detail in two textbooks [8, 30], including numerous examples. The explicit form of the supercurrent conservation equation depends on the off-shell supergravity realization chosen. In the case of the old minimal $(n=-1 / 3)$ formulation for $\mathcal{N}=1$ supergravity [31] (see also [32]), the conservation equation is

$$
\bar{D}^{\dot{\alpha}} J_{\alpha \dot{\alpha}}=D_{\alpha} T, \quad \bar{D}_{\dot{\alpha}} T=0,
$$

with $T$ called the supertrace. In the case of the new minimal $(n=0)$ formulation for $\mathcal{N}=1$ supergravity [33], the conservation equation is

$$
\bar{D}^{\dot{\alpha}} \mathbb{J}_{\alpha \dot{\alpha}}=\mathbb{T}_{\alpha}, \quad \bar{D}_{\dot{\alpha}} \mathbb{T}_{\alpha}=0, \quad D^{\alpha} \mathbb{T}_{\alpha}=\bar{D}_{\dot{\alpha}} \overline{\mathbb{T}}^{\dot{\alpha}} .
$$

The difference between (42) and (43) is due to the different types of compensators used in these supergravity formulations. One can construct more general supercurrent conservation equations, see, e.g., [34, 35].

We wish to analyze the two versions of the supercurrent for the theory (24). Within the old minimal formulation for $\mathcal{N}=1$ supergravity, the supercurrent for the model (21) was computed in [36] and shown to be duality invariant. The supercurrent obtained is rather complicated to deal with. So for simplicity, we restrict our consideration to the Maxwell case, by setting $\Lambda=0$ in (21), similarly to [1]. The consideration below can naturally be generalized to the case $\Lambda \neq 0$.

Using the old minimal formulation for $\mathcal{N}=1$ supergravity, the supermultiplets $J_{\alpha \dot{\alpha}}$ and $T$ are computed to be [1]:

$$
J_{\alpha \dot{\alpha}}=2 W_{\alpha} \bar{W}_{\dot{\alpha}}+\frac{2}{3} \xi\left[D_{\alpha}, \bar{D}_{\dot{\alpha}}\right] V, \quad T=\frac{1}{3} \xi \bar{D}^{2} V .
$$


Using the equation of motion, $D^{\alpha} W_{\alpha}=2 \xi$, one can check that the conservation equation (42) holds. As pointed out by Komargodski and Seiberg [1], both $J_{\alpha \dot{\alpha}}$ and $T$ are not gauge invariant. The reason for this is very simple: minimal coupling of the FI term to supergravity, which makes use of the first expression in (17), is not gauge invariant. Old minimal supergravity is not well suited to describe FI terms.

Using the new minimal formulation for $\mathcal{N}=1$ supergravity, the supermultiplets $\mathbb{J}_{\alpha \dot{\alpha}}$ and $\mathbb{T}_{\alpha}$ can be computed to be:

$$
\mathbb{J}_{\alpha \dot{\alpha}}=2 W_{\alpha} \bar{W}_{\dot{\alpha}}, \quad \mathbb{T}_{\alpha}=4 \xi W_{\alpha} .
$$

Both objects are gauge invariant. The reason for this is very simple: minimal coupling of the FI term to supergravity, which makes use of the second expression in (7) and is obtained by replacing (the vacuum expectation value) $\theta^{\alpha}$ with the chiral spinor compensator, is gauge invariant. New minimal supergravity is ideal for describing FI terms.

The theory (24) is $R$-invariant, and the corresponding $R$-current $j_{\alpha \dot{\alpha}}^{(5)}$ must be conserved. If one identifies $j_{\alpha \dot{\alpha}}^{(5)}$ with the lowest component of the supercurrent in (44), it fails to be conserved, for one finds

$$
\partial^{\alpha \dot{\alpha}} J_{\alpha \dot{\alpha}}=\frac{\dot{\mathrm{i}}}{6} \xi\left[D^{2}, \bar{D}^{2}\right] V \equiv \frac{2}{3} \xi \partial^{\alpha \dot{\alpha}}\left[D_{\alpha}, \bar{D}_{\dot{\alpha}}\right] V \neq 0 .
$$

This result was interpreted in [2] as non-existence of supercurrent for rigid supersymmetric gauge theories with non-zero FI terms. However, if one defines the $R$-current by

$$
j_{\alpha \dot{\alpha}}^{(5)}:=\left.\left(J_{\alpha \dot{\alpha}}-\frac{2}{3} \xi\left[D_{\alpha}, \bar{D}_{\dot{\alpha}}\right] V\right)\right|_{\theta=0},
$$

then it is clearly conserved.

In the case of the supercurrent (45), the standard definition of the $R$-current applies

$$
j_{\alpha \dot{\alpha}}^{(5)}:=\left.\mathbb{J}_{\alpha \dot{\alpha}}\right|_{\theta=0}
$$

Our consideration shows that the supercurrent does exist for globally supersymmetric gauge theories in the presence of FI terms.

\section{Acknowledgements:}

The author is grateful to Ian McArthur and Arkady Tseytlin for useful comments. This work is supported in part by the Australian Research Council.

Note added. The published version of [2], Ref. [37], significantly differs from the original preprint, Ref. [2], discussed above. The conclusions of [37] are similar to those given in the present paper. 


\section{References}

[1] Z. Komargodski and N. Seiberg, JHEP 0906, 007 (2009) arXiv:0904.1159 [hep-th]].

[2] K. R. Dienes and B. Thomas, arXiv:0911.0677v1 [hep-th].

[3] S. Ferrara and B. Zumino, Nucl. Phys. B 87, 207 (1975).

[4] P. Fayet and J. Iliopoulos, Phys. Lett. B 51, 461 (1974).

[5] J. Bagger and A. Galperin, Phys. Rev. D 55, 1091 (1997) arXiv:hep-th/9608177.

[6] M. Roček and A. A. Tseytlin, Phys. Rev. D 59, 106001 (1999) arXiv:hep-th/9811232.

[7] J. Wess and J. Bagger, Supersymmetry and Supergravity, Princeton University Press, Princeton, 1992.

[8] I. L. Buchbinder and S. M. Kuzenko, Ideas and Methods of Supersymmetry and Supergravity or a Walk Through Superspace, IOP, Bristol, 1998.

[9] S. Deser and R. Puzalowski, J. Phys. A 13, 2501 ( 1980).

[10] S. Cecotti and S. Ferrara, Phys. Lett. B 187, 335 (1987).

[11] M. Born and L. Infeld, Proc. Roy. Soc. Lond. A 144, 425 (1934).

[12] I. Antoniadis, J. P. Derendinger and T. Maillard, Nucl. Phys. B 808, 53 (2009) arXiv:0804.1738 [hep-th]].

[13] P. Fayet, Nucl. Phys. B 113, 135 (1976).

[14] I. Antoniadis, H. Partouche and T. R. Taylor, Phys. Lett. B 372, 83 (1996) arXiv:hep-th/9512006.

[15] E. A. Ivanov and B. M. Zupnik, Phys. Atom. Nucl. 62, 1043 (1999) arXiv:hep-th/9710236.

[16] K. Fujiwara, H. Itoyama and M. Sakaguchi, Prog. Theor. Phys. 113 (2005) 429 arXiv:hep-th/0409060; Nucl. Phys. B 723 (2005) 33 arXiv:hep-th/0503113; Nucl. Phys. B 740 (2006) 58 arXiv:hep-th/0510255]; H. Itoyama, K. Maruyoshi and M. Sakaguchi, Nucl. Phys. B 794 (2008) 216 [arXiv:0709.3166 [hep-th]].

[17] D. Brace, B. Morariu and B. Zumino, in The Many Faces of the Superworld: Yuri Golfand Memorial Volume, M. A. Shifman (Ed.), World Scientific, Singapore, 1999, p. 103 arXiv:hep-th/9905218.

[18] S. M. Kuzenko and S. Theisen, JHEP 0003, 034 (2000) arXiv:hep-th/0001068; Fortsch. Phys. 49, 273 (2001) arXiv:hep-th/0007231. 
[19] S. M. Kuzenko and S. A. McCarthy, JHEP 0505, 012 (2005) arXiv:hep-th/0501172].

[20] D. V. Volkov and V. P. Akulov, JETP Lett. 16, 438 (1972); Phys. Lett. B 46, 109 (1973); Theor. Math. Phys. 18, 28 (1974).

[21] T. Hatanaka and S. V. Ketov, Phys. Lett. B 580, 265 (2004) arXiv:hep-th/0310152.

[22] M. K. Gaillard and B. Zumino, Nucl. Phys. B 193, 221 (1981).

[23] G. W. Gibbons and D. A. Rasheed, Nucl. Phys. B454, 185 (1995) 185 arXiv:hep-th/9506035.

[24] M. K. Gaillard and B. Zumino, in Supersymmetry and Quantum Field Theory, J. Wess and V. P. Akulov (Eds.), Springer Verlag, 1998, p. 121 arXiv:hep-th/9705226.

[25] P. Aschieri, S. Ferrara and B. Zumino, Riv. Nuovo Cim. 31, 625 (2009) arXiv:0807.4039 [hep-th]].

[26] G. W. Gibbons and D. A. Rasheed, Phys. Lett. B365 (1996) 46 [arXiv:hep-th/9509141].

[27] A. A. Tseytlin, Nucl. Phys. B469 (1996) 51 arXiv:hep-th/9602064]; M. B. Green and M. Gutperle, Phys. Lett. B377 (1996) 28 arXiv:hep-th/9602077].

[28] T. Kimura and I. Oda, Int. J. Mod. Phys. A 16, 503 (2001) arXiv:hep-th/9904019.

[29] V. Ogievetsky and E. Sokatchev, Nucl. Phys. B 124, 309 (1977).

[30] S. J. Gates Jr., M. T. Grisaru, M. Roček and W. Siegel, Superspace, or One Thousand and One Lessons in Supersymmetry, Benjamin/Cummings (Reading, MA), 1983, hep-th/0108200.

[31] J. Wess and B. Zumino, Phys. Lett. B 74, 51 (1978); K. S. Stelle and P. C. West, Phys. Lett. B 74, 330 (1978); S. Ferrara and P. van Nieuwenhuizen, Phys. Lett. B 74, 333 (1978).

[32] W. Siegel, Nucl. Phys. B 142, 301 (1978); W. Siegel and S. J. Gates Jr. Nucl. Phys. B 147, 77 (1979).

[33] V. P. Akulov, D. V. Volkov and V. A. Soroka, Theor. Math. Phys. 31, 285 (1977);

M. F. Sohnius and P. C. West, Phys. Lett. B 105, 353 (1981).

[34] O. Piguet and K. Sibold, Renormalized Supersymmetry: The Perturbation Theory of N=1 Supersymmetric Theories in Flat Space-Time, Birkhäuser, Boston, 1986.

[35] T. E. Clark and S. T. Love, Int. J. Mod. Phys. A 11, 2807 (1996) arXiv:hep-th/9506145.

[36] S. M. Kuzenko and S. A. McCarthy, JHEP 0302, 038 (2003) arXiv:hep-th/0212039].

[37] K. R. Dienes and B. Thomas, Phys. Rev. D 81, 065023 (2010). 